\title{
Novel Transform Domain Techniques for Person Identification using ECG Signals
}

\author{
Rudresh M. D. \\ Research Scholar, JNTU, \\ Hyderabad \\ Kalpataru Institute of Technology \\ Tiptur, Karnataka, India
}

\author{
H. S. Jayanna \\ Professor, Dept. of ISE \\ Siddaganga Institute of \\ Technology \\ Tumkur, Karnataka, India
}

\author{
Anitha Sheela K. \\ Professor, Dept. of ECE \\ JNTU, Hyderabad \\ Telangana State, India
}

\begin{abstract}
The person identification is an active area in research fields. many person identification techniques have been proposed in literature both in time domain and transformed domain. An improved various transformed domain techniques are proposed in this paper. This paper work also demonstrates the task of identifying the person with the various segments of ECG signals, and also investigates which segments of ECG signals has more person specific information by using transformational methods. A transformed domain technique includes discrete fourier transform, discrete cosine transform and discrete wavelet transform. An experimental results on ECG signals using transformed domain techniques demonstrates that the improvement of proposed techniques over those of time domain techniques.
\end{abstract}

\section{Keywords}

ECG person identification, CCORR, PRD, Wavlet distance measure.

\section{INTRODUCTION}

In today's world, we can see the application of person recognition in many places starting from commercial applications, such as internet access, ATM, credit card, cellular phone, to the government and forensic applications like driver's license, pass-port control and criminal investigation, terrorist identification etc, The main purpose of of person identification system is to ensure that the rendered services are accessed only by a legitimate user and no one else, i.e., to confirm or determine the identity of an individual requesting for service.

Traditional password-based and other identification systems have a number of flaws. A password can be forgotten or elicited, and such systems are easy to hack. One needs to always carry the identification key around, which is inconvenient. there are chances that the intruder can get hold of your password. Besides, a person cannot be identified without any specific physical carrier. Together, all those factors prompt us to look for new approaches to the problem. Biometrics techniques truly identifies the actual applicant as a particular individual than other traditional methods like photo identity card, tokens, badges and passwords etc which may be hacked by somebody. Biometrics provides an automatic method of recognizing a person based upon intrinsic physical or behavioral features, such as face, voice, retinal structure, gait, finger print, etc [10-14]. Scientists are coming with many new techniques to make robust system for person recognition; imposters are also Imposters are also com- -ing with techniques to fool the existing system take advantage of that system. In existing person identification systems, we use Fingerprint is the most widespread biometric, it has been used in forensic for about 100 years. An imposter can fool a finger print system by recreating in latex, similarly can fool the face recognition system by providing photo and can also fool voice recognition by imitating other voice. The possibility to steal data directly from subjects and the relative ease to replicate them (e.g. silicone fingerprints, pictures or facial masks) posed serious challenges to researchers and multimodal recognition systems have been suggested.

\subsection{Literature survey}

In recent years, the Electrocardiogram (ECG) has been proposed as novel biometric for person identification, dedicated research on the ECG analysis has demonstrated its advantages in biometrics: ECG is present in all living individuals, exhibits the typical characteristics of a biometric and it is hard to forge. In addition to this, ECG analysis is a robust method to detect the liveness (the person has to present) characteristic of the subject in authentication scenarios. This special characteristic of ECG helps the system not to be make fool by imposter. ECG is a method to measure and record electrical potential generated by the heart.

The hypothesis of the use of ECG as personal identification attribute was suggested by Scientist Forsen G E[1] ; however, the first study on the ECG analysis for biometric purposes was carried out by Biel and colleagues: the authors investigated some combinations of features in 12-leads ECG recordings on a sample of 20 subjects[2][3].

In [8], it is suggested that variability in heart rate could also another measure useful in person identification. ECG data were recorded under several physiological states. To distinguish subjects, the author utilized features including the mean and variance of the R-R interval, along with other ECG parameters such as width of $\mathrm{P}, \mathrm{R}$, and $\mathrm{T}$ waves. This modality of using ECG data in person identification offers some unique advantages. It is proposed that ECG person identification would be particularly effective in health care applications as the ECG is frequently used to monitor a patient's condition[3],[5]; therefore, without any additional data requirements, this can be used to verify a patient's identity in medical records or other medical procedures.

In [9], ECG data were used as one of source of biometric data along with the facial image data. Supplementing other biometrics with ECG information can be accomplished with little user perceived change in the interface. For example, the ECG data can be recorded simultaneously in fingerprint and 
palm recognition systems. Multimodal person identification techniques can provide can provide significant increases in performance as monomodal methods begin to saturate in their performance [12], ECG information would provide a method for liveness detection, increasing system reliability.

In[my] paper research work study investigates the feasibility of using the Electrocardiogram (ECG) complexes as a new biometric features for human identification and verification.ECG waveforms of different person are different but it is unclear whether such differences can be used identify different individuals. This research work successfully demonstrated that it is possible to identify a specific person from group of persons by segmented ECG signal. The person specific features of ECG signal can be verified by collecting ECG data of 5 individuals of different sessions, drawing time domain, frequency domain and cepstral domain plots results shown that ECG is used for biometric.

In this paper research work, we investigate the influence of intra subject variability by collecting data from 30 subjects in two different sessions with an interval of 15days unlike the previous study. and then, to see which part of ECG complex has more person specific information, the ECG signal has further segmented and performance is evaluated by using both time domain and transformed domain techniques. The duration and sampling rate of data's are 60 seconds and $8 \mathrm{kHz}$ respectively.

Our study is organized as follows: Section $\mathrm{I}$ is the introduction, Section II describes a physiological study of ECG, Section III describes the methods of person identification using ECG. Section IV presents Experimental Results. Finally, the conclusion is made in Section V.

\section{$1.2 \quad$ Electrocardiogram}

The Electrocardiogram (ECG) is graphical recording of the electrical activity of the heart. The ECG records cardiac currents by means of metal electrodes placed on the surface of the body. When the cardiac impulse passes through the heart, electrical current also spreads from the heart into the adjacent tissues surrounding the heart. A small portion of the current spreads all the way to the surface of the body. If electrodes are placed on the skin on opposite sides of the heart, electrical potentials generated by the current can be recorded; the recording is known as an electrocardiogram.

\subsection{Characteristics of the Normal ECG}

The normal electrocardiogram shown in Figure 1 is composed of a $\mathrm{P}$ wave, a QRS complex, and a $\mathrm{T}$ wave. The QRS complex is often, but not always, three separate waves: the Q wave, the $R$ wave, and the $S$ wave. The $P$ wave is caused by electrical potentials generated when the atria depolarize before atria contraction begins. The QRS complex is caused by potentials generated when the ventricles depolarize before contraction, that is, as the depolarization wave spreads through the ventricles. Therefore, both the $\mathrm{P}$ wave and the components of the QRS complex are depolarization waves. The $T$ wave is caused by potentials generated as the ventricles recover from the state of depolarization. This process normally occurs in ventricular muscle 0.25 to 0.35 second after depolarization, and the $\mathrm{T}$ wave is known as a repolarization wave. Thus, the electrocardiogram is composed of both depolarization and repolarization waves. The distinction between depolarization waves and repolarization waves is so important in electrocardiography that further $\mathrm{C}$

\subsection{Normal Voltages in the Electro}

\section{Cardiogram}

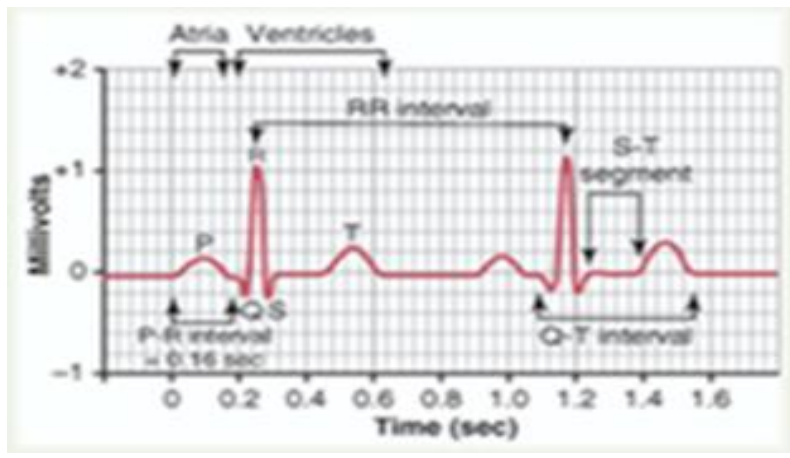

The recorded voltages of the waves in the normal electrocardiogram depend on the manner in which the electrodes are applied to the surface of the body and how close the electrodes are to the heart. When one electrode is placed directly over the ventricles and a second electrode is placed elsewhere on the body remote from the heart, the voltage of the QRS complex may be as great as 3 to 4 millivolts. Even this voltage is small in comparison with the monophasic action potential of 110 millivolts recorded directly at the heart muscle membrane. When electrocardiograms are recorded from electrodes on the two arms or on one arm and one leg, the voltage of the QRS complex usually is 1.0 to 1.5 millivolt from the top of the $\mathrm{R}$ wave to the bottom of the $\mathrm{S}$ wave; the voltage of the $\mathrm{P}$ wave is between 0.1 and 0.3 millivolt; and that of the $T$ wave is between 0.2 and 0.3 millivolt. The time between the beginning of the $\mathrm{P}$ wave and the beginning of the QRS complex is the interval between the beginning of electrical excitation of the atria and the beginning of excitation of the ventricles. This period is called the $\mathrm{P}-\mathrm{Q}$ interval. The normal $\mathrm{P}-\mathrm{Q}$ interval is about 0.16 second. (Often this interval is called the P-R interval because the $\mathrm{Q}$ wave is likely to be absent. Contraction of the ventricle lasts almost from the beginning of the $\mathrm{Q}$ wave (or $\mathrm{R}$ wave, if the $\mathrm{Q}$ wave is absent) to the end of the $\mathrm{T}$ wave. This interval is called the Q-T interval and ordinarily is about 0.35 second.

\section{METHODS OF PERSON IDENT I - FICATION USING ECG}

Direct and transformational methods are used for implementation of person identification system using ECG. The direct methods such as PRD (Percent Residue Difference) and CCORR (Correlation Co-efficient) process the signal in time domain. Whereas the transformational methods such as DCT(Discrete Cosine Transform) and wavelet transform process the signal in the transformed domain. The two methods used for identification of person by using ECG are:

\section{$>\quad \mathrm{PRD}($ Percent Residual Difference) \\ $>\operatorname{CCORR}($ Correlation Co-efficient)}

These methods are so called because they process the signal in time domain itself.

The PRD is a common quantitative measure, which is used for evaluation of difference between two signals. It is defined as

$$
\boldsymbol{P R D _ { n }}=\sqrt{\frac{\sum_{i=1}^{M}\left(\boldsymbol{x}_{\mathbf{0}}(i)-\boldsymbol{x}_{\boldsymbol{n}}(i)\right)^{2}}{\sum_{j=1}^{M}\left(\boldsymbol{x}_{\mathbf{0}}(j)-\overline{\boldsymbol{x}_{\mathbf{0}}}(j)\right)^{2}}} \times 100
$$


Where $\boldsymbol{x}_{\mathbf{0}}$ is the unknown ECG complex and $\boldsymbol{x}_{\boldsymbol{n}}$ is the enrolled ECG complex for subject $n(n=1,2,----\mathrm{N}), \mathrm{N}$ is the number of person enrolled in data base.

In PRD the energy of difference between two signals, i.e., unknown ECG complex and enrolled ECG data is calculated and normalized by the energy of the zero mean of unknown ECG complex. For the PRD measures the person associated with the enrolled data with the lowest PRD is selected as a match.

The CCORR quantifies a linear least squares fitting between two data sets and is computed as

$$
\operatorname{CCORR}_{n}=\frac{\sum_{i=1}^{M}\left(x_{0}(i)-\overline{x_{0}}\right)\left(x_{n}(i)-\overline{x_{n}}\right)}{\sqrt{\sum_{i=1}^{M}\left(x_{0}(i)-\overline{x_{0}}\right)^{2} \sum_{i=1}^{M}\left(x_{n}(i)-\overline{x_{n}}\right)^{2}}}
$$

Where $\mathbf{X}_{\mathbf{0}}$ is the unknown ECG signal and $\boldsymbol{x}_{\boldsymbol{n}}$ is the enrolled ECG signal for subject $\mathrm{n}(\mathrm{n}=1,2 \ldots \mathrm{N} ; \mathrm{N}$ is the number of person enrolled in the data base and $\overline{\boldsymbol{x}_{\mathbf{0}}}$ and $\overline{\boldsymbol{x}_{\boldsymbol{n}}}$ are the mean values of $\mathbf{x}_{\mathbf{0}}$ and $\boldsymbol{x}_{\boldsymbol{n}}$ respectively. For the CCORR measure, the person associated with the enrolled data with the highest $C C O R R$ value is selected as a match.

\subsection{Transformational}

To further verify the variability of the ECG signals, the transformational methods are used, they are

\section{$>$ DFT (Discrete Fourier Transform) \\ $>$ DCT (Discrete Cosine Transform) \\ $>$ Wavelet transforms.}

These methods are so called because they process the signal in the transformed domain or frequency domain.

\subsection{Discrete Fourier Transform}

A DFT decomposes a sequence of values into component of different frequencies. Let $\mathrm{x}_{0}, \mathrm{x}_{1} \ldots \ldots \mathrm{x}_{\mathrm{n}-1}$ be the complex numbers.

The DFT is defined by the formula

$$
\boldsymbol{X}(\boldsymbol{K})=\sum_{n=0}^{N-1} \boldsymbol{x}(\boldsymbol{n}) e^{-j \frac{2 \pi}{N} k n}
$$

DFT is efficiently computed by Fast Fourier Transform (FFT), it is a efficient method of computing DFT with reduced number of calculations. Here, the DFT coefficients are computed for test ECG data and Euclidean distance is measured between DFT coefficients of test data and DFT coefficients of stored database data. For the DFT measure, the person associated with the enrolled data with the lowest Euclidean distance is selected as match.

\subsection{Discrete Cosine Transform}

A discrete cosine transform (DCT) express a sequence of finitely many data points in terms of a sum of cosine functions oscillating at different frequencies. DCT is important to numerous applications in science and engineering, from lossy compression of audio and images (where small high frequency components can be discarded), to spectral methods for the numerical solutions of partial differential equations. The uses of cosine rather than sine function are much more efficient, whereas for differential equation, the cosines function express a particular choice of boundary conditions. The most common variant of discrete cosine transform is the type-II DCT. This is often called simply "The DCT", its inverse, the type-III DCT, is correspondingly often called simply "The inverse DCT" or "The IDCT". defined as

The type-II N-point discrete cosine transform is

$$
\boldsymbol{X}_{\boldsymbol{D C T}[\boldsymbol{K}]}=\sum_{n=0}^{N-1} \boldsymbol{2} \boldsymbol{X}(\boldsymbol{n}) \cos \left(\frac{\pi k(2 n+1)}{2 n}\right)_{\mathbf{0} \leq \boldsymbol{k} \leq \boldsymbol{N}-\mathbf{1}}
$$

\subsection{Wavelet distance measure}

Wavelet analysis allows the use of long time intervals where we want more precise low frequency information and shorter regions where we want high frequency information. One major advantage afforded by wavelets is the ability to perform local analysis i.e. to analyze the localized area of larger signal. wavelet analysis is capable of revealing aspects of data that other signal analysis techniques miss, aspects like trends, breakdown points, discontinuities in higher derivatives, and self-similarity. Furthermore because it affords a different view of data than those presented by traditional techniques, wavelet analysis can often compress or de noises a signal without appreciable degradation. In[13] wavelet analysis, we often speak of approximation and details. The approximations are the high scale, low frequency components of the signal. The details are the low scale, high frequency components. Detail coefficients of the discrete wavelet transform $\gamma^{p, q}$ detail coefficient $\mathrm{q}$ from the $\mathrm{P}^{\text {th }}$ level of decomposition and approximation coefficients $\boldsymbol{\delta}$ are computed for each signal using these coefficients a distance is computed as

$$
\boldsymbol{W D I S T}_{\boldsymbol{n}}=\sum_{P=1}^{P} \sum_{q=1}^{Q} \frac{\left|\left(\gamma_{0}^{p, q}+\delta\right)-\left(\gamma_{n}^{p, q}+\delta\right)\right|}{\max \left|\left(\gamma_{0}^{p, q}+\delta\right)\right|}
$$

The numerator is the absolute difference of the wavelet coefficients from the unknown signal $\mathrm{x}_{0}$ and the enrolled data $\mathrm{x}_{\mathrm{n}}$. The contribution of this difference is based upon the relative amplitude of the wavelet coefficient from the unknown signal. For the WDIST measure, the person associated with the enrolled data with the lowest WDIST is selected as a match.

Three different techniques are used to evaluate the performance of ECG based person identification system using above mentioned methods. These are named as

$$
\begin{array}{ll}
> & \text { Many-many } \\
> & \text { Many-one } \\
> & \text { One-one }
\end{array}
$$

In many-many, different ECG complexes of an individual is compared with the data stored in the data base. Where all ECG complexes of every individual are stored.

In the case of many-one, an average ECG complex of all individual use stored in the data base and during testing. Different ECG complexes of an individual are compared with all averaged ECG data. In one-one, the averaged ECG complex of an individual is compared with the average ECG complexes of all individual stored in data base. 


\section{EXPERIMENTAL RESULTS}

The techniques which are discussed in previous sections are used for analysis of PQRST complex of ECG signal. To see which part of ECG signal complex has person specific information, ECG complexes are further segmented and Pwave, QRS complex and T-wave are extracted. Since techniques which already used for PQRST complexes to get performances are used for the segmented signals and the performance is summarized in the below table.

Table1, 2, 3, 4 and 5 show the ECG person identification performances of, DFT, DCT and Wavelet measure methods. Performance of different complexes of ECG, i.e., PQRST, P, QRS and T-wave are shown in column. Each column is further subdivided into two parts. i.e., $\mathrm{CC}$ and $\mathrm{MC}$, where $\boldsymbol{C C}$ refers to number of subjects correctly classified and $M C$ refers to number of subjects miss classified. Total 30subjects data are tested. Number of subjects correctly identified or miss classified are shown by CC and MC. Performance of manymany, many-one and one-one techniques are shown in rows. From above results of various methods and techniques, we can easily see that, P-wave and QRS-wave generally have more information about the person than $\mathrm{T}$-wave.

The QRS complex has highest information about the person and the T-wave has least person related information, as Twave has low frequency, it is believe that with heart rate variability the shape of T-wave changes which results poor performance.

Table 1. Performance table of DFT

\begin{tabular}{|c|c|c|c|c|c|c|c|c|}
\hline \multirow{2}{*}{ Wave } & \multicolumn{2}{|c|}{ PQRST } & \multicolumn{2}{|c|}{ P } & \multicolumn{2}{|c|}{ QRS } & \multicolumn{2}{|c|}{ T } \\
\cline { 2 - 10 } & CC & MC & CC & MC & CC & MC & CC & MC \\
\hline Many-many & 22 & 08 & 19 & 11 & 22 & 08 & 16 & 14 \\
\hline Many-one & 21 & 09 & 19 & 11 & 19 & 11 & 17 & 13 \\
\hline One-one & 21 & 09 & 19 & 11 & 20 & 10 & 16 & 14 \\
\hline
\end{tabular}

Table 2. Performance table of DCT

\begin{tabular}{|c|c|c|c|c|c|c|c|c|}
\hline \multirow{2}{*}{ Wave } & \multicolumn{2}{|c|}{ PQRST } & \multicolumn{2}{|c|}{ P } & \multicolumn{2}{c|}{ QRS } & \multicolumn{2}{|c|}{ T } \\
\cline { 2 - 10 } & CC & MC & CC & MC & CC & MC & CC & MC \\
\hline Many-many & 24 & 06 & 22 & 08 & 24 & 06 & 13 & 17 \\
\hline Many-one & 22 & 08 & 22 & 08 & 20 & 10 & 12 & 18 \\
\hline One-one & 23 & 07 & 22 & 08 & 21 & 09 & 11 & 19 \\
\hline
\end{tabular}

Table 3. Performance table of Wavelet Measure

\begin{tabular}{|c|c|c|c|c|c|c|c|c|}
\hline \multirow{2}{*}{ Wave } & \multicolumn{2}{|c|}{ PQRST } & \multicolumn{2}{|c|}{ P } & \multicolumn{2}{|c|}{ QRS } & \multicolumn{2}{|c|}{ T } \\
\cline { 2 - 10 } & CC & MC & CC & MC & CC & MC & CC & MC \\
\hline Many-many & 24 & 06 & 21 & 09 & 23 & 07 & 15 & 15 \\
\hline Many-one & 24 & 06 & 20 & 10 & 23 & 07 & 14 & 16 \\
\hline One-one & 24 & 06 & 22 & 08 & 22 & 08 & 13 & 17 \\
\hline
\end{tabular}

Table 4. Performance table of Wavelet coefficients

\begin{tabular}{|c|c|c|c|c|c|c|}
\hline \multirow{2}{*}{$\begin{array}{c}\text { Approximate and } \\
\text { details coefficients }\end{array}$} & \multicolumn{2}{|c|}{ Many-many } & \multicolumn{2}{c|}{ Many-one } & \multicolumn{2}{c|}{ One -one } \\
\cline { 2 - 7 } & $\mathbf{C C}$ & MC & CC & MC & CC & MC \\
\hline $1-31.125 \mathrm{~Hz}(\mathrm{~A} 5)$ & 24 & 06 & 22 & 08 & 23 & 07 \\
\hline $31.125-62,25 \mathrm{~Hz}(\mathrm{D} 5)$ & 25 & 05 & 25 & 05 & 25 & 05 \\
\hline $62.25-125 \mathrm{~Hz}(\mathrm{D} 4)$ & 20 & 10 & 25 & 05 & 25 & 05 \\
\hline $125-250 \mathrm{~Hz}(\mathrm{D} 3)$ & 16 & 14 & 27 & 03 & 25 & 05 \\
\hline $250-500 \mathrm{~Hz}(\mathrm{D} 2)$ & 15 & 15 & 24 & 06 & 25 & 05 \\
\hline $500-1000 \mathrm{~Hz}(\mathrm{D} 1)$ & 10 & 20 & 15 & 15 & 12 & 18 \\
\hline
\end{tabular}

Table 5. Performance table of Wavelet coefficients (approximate+details)

\begin{tabular}{|c|c|c|c|c|c|c|}
\hline $\begin{array}{c}\text { Approximate and } \\
\text { details coefficients }\end{array}$ & \multicolumn{2}{|c|}{$\begin{array}{c}\text { Many- } \\
\text { many }\end{array}$} & \multicolumn{2}{|c|}{ Many-one } & \multicolumn{2}{c|}{ One -one } \\
\cline { 2 - 7 } & CC & MC & CC & MC & CC & MC \\
\hline $\begin{array}{c}1-31.125 H z \\
(A 5)\end{array}$ & 24 & 06 & 22 & 08 & 23 & 07 \\
\hline $\begin{array}{c}31.125-62,25 H z \\
(A 5+D 5)\end{array}$ & 25 & 05 & 24 & 06 & 24 & 06 \\
\hline $\begin{array}{c}62.25-125 H z \\
(A 5+D 5+D 4)\end{array}$ & 24 & 06 & 24 & 06 & 24 & 06 \\
\hline $\begin{array}{c}125-250 H z \\
(A 5+D 5+D 4+D 3)\end{array}$ & 24 & 06 & 24 & 06 & 24 & 06 \\
\hline $\begin{array}{c}250-500 H z \\
(A 5+D 5+D 4+D 3+D 2)\end{array}$ & 24 & 06 & 24 & 06 & 24 & 06 \\
\hline $\begin{array}{c}500-1000 H z(D 1) \\
(A 5+D 5+D 4+D 3+D 2)\end{array}$ & 24 & 06 & 24 & 06 & 24 & 06 \\
\hline
\end{tabular}

The better performance of QRS-wave is expected because of its highest frequency component which does not vary with heart rate variability.

Among the three techniques, many-many has higher performance than many-one and one-one. It has been seen the probability of false recognition in many-many technique is lower than many-one and one-one techniques.

In other word, system makes a hasty decision in many-one and one-one, where as in many-many. If same decision is not in favor of few complexes still the system has a chance to identify the legitimate person.

To see the person related information in each level in wavelet domain, approximate and detail coefficients are calculated and performances are evaluated. Table 4 shows the performances using approximate and details coefficients of each level. The Performances using Techniques, i.e. many-many, many-one, one-one, are shown in columns. As frequency of ECG lies in the range of $3-50 \mathrm{~Hz}$, it was expected that only approximate and detail 1 will give better performances and rest will give poor performances, which can be observed in many-many techniques for approximate and details coefficients. But, some aberrant results also observed. For example, in many-one technique the $\mathrm{CC}$ values does not decrease and, in one-one, $\mathrm{CC}$ and $\mathrm{MC}$ are remain constant. To see how much information each detail coefficient level adds to the performance, a new measure is implanted where every time next detail level is added with the approximated coefficients and performances are evaluated. Table 5 shows the performances. As shown in First column A1 (Approximate coefficients) is added with detail coefficients in each level and corresponding results are shown in the columns. Which shows that in all the three techniques only approximate and detail coefficients of the first level has most information about the person and others have least, as the result the performances does not change when higher level coefficients are considered. 


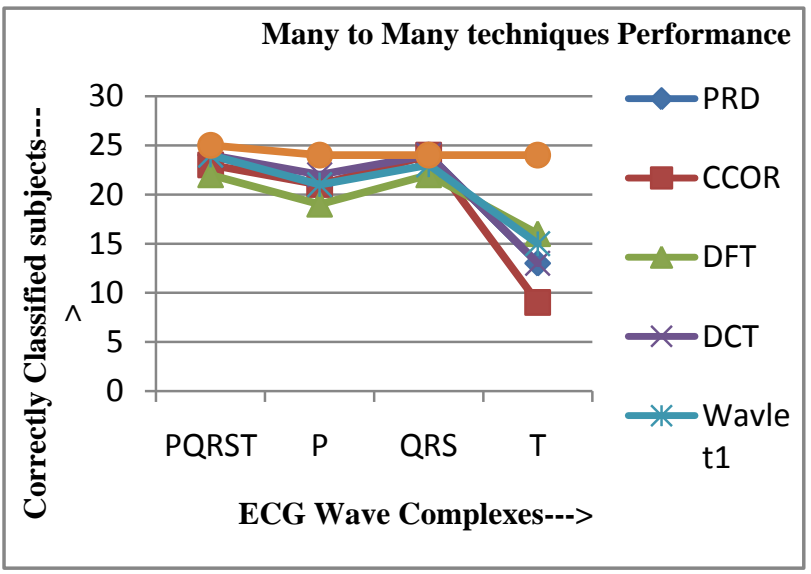

Fig 2: Many to Many Technique Performances

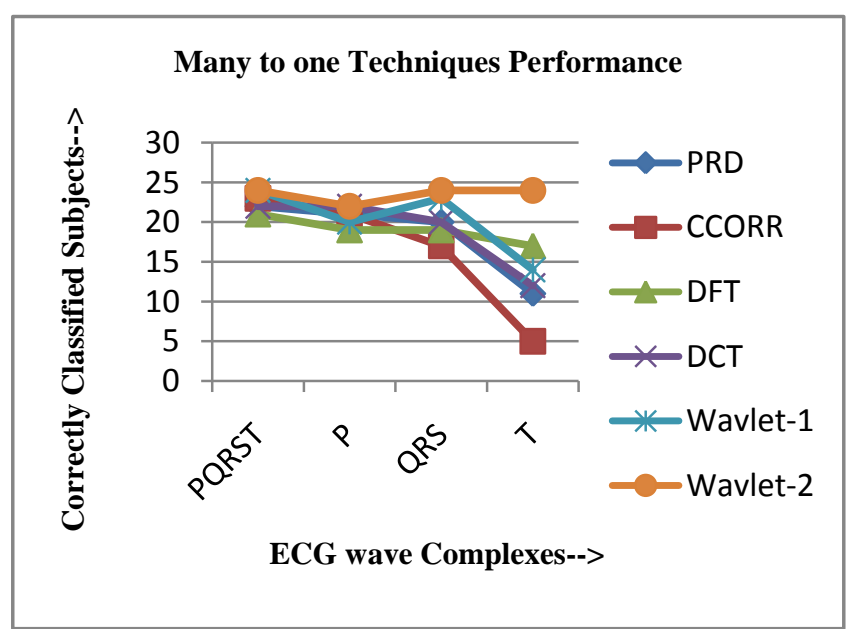

Fig 3: Many to One Technique Performances

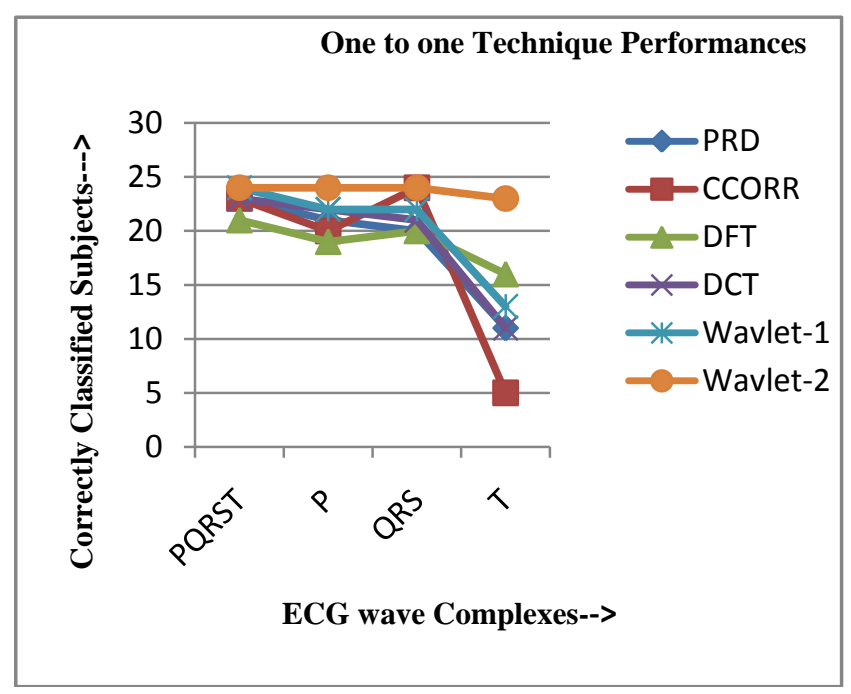

Fig 4: One to One Technique Performances

\section{CONCLUSION}

In this paper research work, the feasibility of ECG person identification using transform domain technique has been demonstrated by empirical results. A new classification methodology was introduced using a distance measurement based on wavelet coefficients for the data of different segmented ECG complexes; this wavelet distance measure outperformed the CCORR, PRD and other transformable approaches. This results shows that the ECG data provide the same level of uniqueness and stability as conventional biometrics. Hence the ECG can provide excellent source of supplementary data in a multimodal biometric system. And also this study showed that the ECG is not only used in health related applications and verify the identity of a patient prior to drug admiration to avoid medical care errors.

\section{REFERENCES}

[1] L. Biel, O. Pettersson, L. Philipson and P. Wide, "ECG analysis: a new approach in human identification," in IEEE Transactions on Instrumentation and Measurement, vol. 50, no. 3, pp. 808-812, Jun 2001.

[2] M. Kyoso, A. Uchiyama, "Development of an ECG identification system", Proceedings of the 23rd IEEE Engineering and Medicine and Biology Conference, vol. 4, pp. 3721-3723, 2001.

[3] M. Kyoso "A technique for avoiding false acceptance in ECG identification", Proceedings of the IEEE EMBS Asian-Pacific Conference on Biomedical Engineering, pp. 190-191, 2003.

[4] T. W. Shen, W. J. Tompkins, Y. H. Hu, "One-Lead ECG for Identity Verification", Proceedings of the 2nd Joint EMBS/BMES Conference, pp 62-63, 2002.

[5] Jain A K, Ross A, Prabhakar S. An introduction to biometric systems. IEEE Trans Circuits Syst Video Technol. 2004; 14: 4-20.

[6] Jain A K, Hong L, Pankanti S. Biometric identification. Commun ACM. 2000; 43 (2): 91-8.

[7] Flaten GR, Grung B, Kvalheim OM. A method for validation of reference sets in SIMCA modelling. Chemometr Intell Lab Syst. 2004; 72: 101-9.

[8] Hong L, Jain AK. Integrating faces and fingerprints for personal identification. IEEE Trans Pattern Anal Mach Intell. 1998; 20: 1295-307.

[9] Daugman J. Recognizing persons by their Iris patterns. In: Jain AK, Bolle R, Pankanti S, editors. Biometrics: Personal Identification in a Networked Society. New York: Springer Science \& Business Media; 1999. p. 103 21.

[10] Lu G, Zhang D, Wang K. Palmprint recognition using eigenpalms features. Pattern Recognit Lett. 2003; 24: 1463-7.

[11] J. M. Irvine, B. K.Wiederhold, L. W. Gavson, S. Israel, S. B. McGehee, R. Meyer, and M.D. Wiederhold, "Heart rate variability: A new biometric for human identification", in Proc. Int. Conf. Artif. Intell.,2001,pp.1106-1111.

[12] 11. S. A. Israel, W. T. Scruggs, W. J. Worck, J. M. Irvine, "Fusing Face and ECG for Person Identification", Proceedings of the 32nd IEEE Applied Imagery Pattern Recognition Workshop, p. 226, 2003. 
[13] Hashiyada M. Development of biometric DNA ink for authentication security. Tohoku J Exp Med. 2004 Oct; 204 (2): 109-17.

[14] Prokoski FK. Disguise detection and identification using infrared imagery. Proceedings of SPIE 0339, Optics and Images in Law Enforcement II, 27 (June 23, 1983). p. 27-31. doi: 10.1117/12.933650.

[15] Nalwa VS. Automatic on-line signature verification. Proc IEEE. 1997 Feb; 85 (2): 215-39. doi: 10.1109/5.554220.

[16] A. D. C. Chan, M. M. Hamdy, A. Badre and V. Badee, "Wavelet Distance Measure for Person Identification Using Electrocardiograms," in IEEE Transactions on Instrumentation and Measurement, vol. 57, no. 2, pp. 248-253, Feb. 2008.

[17] Choras M. The lip as a biometric. Pattern Anal Appl. 2009; 13 (1): 105-12.

[18] Saini R, Rana N. Comparison of various biometric methods. Int J Adv Sci Technol (IJAST). 2014; 2 (1): 24-30.
[19] Israel SA, Irvine JM, Cheng A, Wiederhold MD, Wiederhold BK. ECG to identify individuals. Pattern Recognit. 2005; 38 (1): 133-42.

[20] Saechia S, Koseeyaporn J. Wardkein P. Human identification system based ECG signal. Proceedings of the Tencon 2005 - 2005 IEEE Region 10; 2005 Nov 21-24; Melbourne, Australia. p. 1-4.

[21] M. S. Islam and N. Alajlan, "An efficient QRS detection method for ECG signal captured from fingers," Multimedia and Expo Workshops (ICMEW), 2013 IEEE International Conference on, San Jose, CA, 2013, pp. $1-5$.

[22] .K. A. Sidek, I. Khalil and H. F. Jelinek, "ECG Biometric with Abnormal Cardiac Conditions in Remote Monitoring System," in IEEE Transactions on Systems, Man, and Cybernetics: Systems, vol. 44, no. 11, pp 1498-1509, Nov. 2014

[23] M. D. Rudresh, H. S. Jayanna and K. A. Sheela, "Person specific characteristic analysis time domain techniques for ECG signals," 2016 IEEE International Conference on Recent Trends in Electronics, Information \& CommunicationTechnology(RTEICTBangalore,2016,pp. 441-446. 